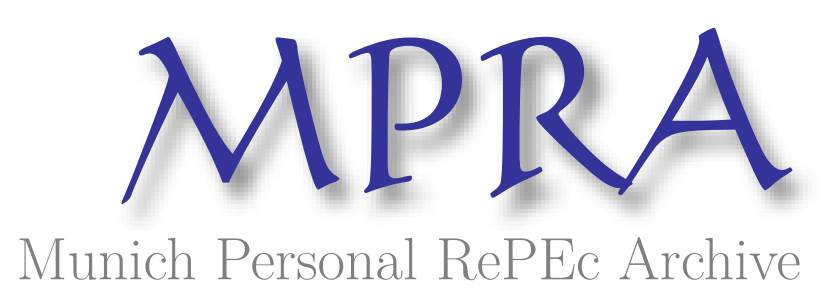

\title{
Optimal Contracting Of New Experience Goods
}

Deb, Rahul

Yale University

4 August 2008

Online at https://mpra.ub.uni-muenchen.de/9880/

MPRA Paper No. 9880, posted 07 Aug 2008 11:36 UTC 


\title{
OPTIMAL CONTRACTING OF NEW EXPERIENCE GOODS
}

\author{
RAHUL DEB ${ }^{\dagger}$
}

\begin{abstract}
We model new experience goods in the context of dynamic mechanism design. These are goods for which an agent is unsure of her valuation but can learn it through consumption experience. We consider a dynamic environment with a single buyer and seller in which contracting occurs over $T$ periods, where each time the agent consumes the object, she receives a signal which allows her to revise her valuation. In this setting, experimentation with the product is strategic both for the buyer and seller. We derive the efficient and seller optimal contracts and compare them. We present a simple two period example which highlights some of the key features of the model. Finally, the methodology developed in the paper can be used to design efficient and optimal contracts in a multi-buyer setting with learning, where each buyer has single unit demand and there is a single object for sale in each period.
\end{abstract}

Keywords: Dynamic mechanism design, new experience goods, bandit problems.

\section{INTRODUCTION}

Mechanism design is perhaps the most prominent method used by economists to design contracts and study price discrimination in a variety of different settings. However, the majority of the literature focuses on static models in which contracting occurs only in a single period and assumes, that the agents know their valuations at the time at which they agree to the contract. Recently, there have been exciting developments which extend some standard results of static mechanism design theory to a general dynamic setting. In dynamic mechanism design, the agents' valuations follow a stochastic process, which implies that they have new private information at each period. The contract offered by the principal in a given period depends not only on the current report by an agent but also on all previous reports as well. Athey and Segal (2007) and Bergemann and Valimaki (2008) study the design of efficient mechanisms in a dynamic context whereas Pavan, Segal and Toikka (2008) (henceforth referred to as PST) derive a seller optimal mechanism for multiperiod contracting, extending the results of Myerson (1981) to a dynamic setting.

This paper studies a multi period contracting problem with learning. We set up a model where an agent would like to contract with the principal for $T$ periods. At each period the agent has single unit demand for the object, the valuation of which evolves over time. If the agent consumes the object at any period, she receives a signal from her consumption experience which allows her to

${ }^{\dagger}$ Department of Economics, Yale University, Box 208268, New Haven, CT 06520-8268

E-mail address: rahul.deb@yale.edu.

Date: August 4, 2008.

I would like to thank Dirk Bergemann for invaluable guidance on this project. I would also like to thank Maher Said for useful comments. Needless to say, all errors are mine. 
update her valuation for the object. This is the key difference between a model of learning and the standard dynamic mechanism design problem. In standard dynamic mechanism design the agent's valuation evolves over time whether or not she consumes the object. In our setting, valuations are only updated by consumption and hence there is a potential benefit to experimentation for the buyer. Moreover, if the seller expects the buyer to like the product after she tries it, she can extract surplus from the buyer for allowing her to experiment and update her valuation. Hence, experimentation affects both the buyer's and seller's incentives and hence social welfare and seller revenue.

This model is relevant to a number of commonly occurring situations. Introductory pricing is a well-known phenomenon where a seller offers a low price initially to the buyer, so that the buyer can learn the attributes of the good. After the introductory pricing period, the seller typically offers a different price to the buyer who may or may not choose to continue consuming the product. An implicit motivation of such behavior is that the buyer may be imperfectly informed or simply unaware about her valuation for the product but by allowing her to experiment for a low price, she can revise her valuation through consumption experience. Such situations are rife in the real world. We observe cable television companies offering discounts for the first few months of service, gyms which allow one to try the equipment for free for the first few weeks and software which offer a free initial trial period following which a license must be purchased to continue using the software. Interestingly, our model predicts that, in general, such pricing is not revenue maximizing.

Specifically, we model a multi-period environment with a single buyer and seller. The buyer has an initial private valuation $\theta_{1}$ for the object which is unobserved by the seller. If the buyer consumes the object in any period $t$, she receives a signal $\xi_{t}$ from her experimentation which allows her to reassess the value of the object and then decide whether to consume it again in the subsequent periods. The signal $\xi_{t}$ is unobserved by the seller. At each period the buyer reports a message to the seller if she has new private information, i.e., a revised valuation for the object. The seller commits to a $T$ period contract at period 1 . This contract offers a menu of prices and probabilities of receiving the object at each period $t$, which depends on not only the period $t$ message (if there is new private information) but also on all previous messages. Lastly, we assume that the buyer can break the contract and walk away at any period. We derive the socially efficient contract and the revenue maximizing contract for the seller.

This problem bears resemblance to the multi-armed bandit problem. The multi-armed bandit problem is a statistical decision model of an agent trying to optimize her decisions while simultaneously improving her information. It can be thought of in terms of a gambler who sequentially chooses which of $K$ different arms of a slot machine to play so as to maximize her reward. Choosing an arm leads to an instantaneous payoff but the process which determines the payoff evolves during the course of play. The key feature is that the distribution of returns from an arm only change when it is chosen. Hence, there is a trade-off between exploring different arms to discover the optimal one and exploiting the arm which is known to give the best payoff at the present time (see Bergemann and Valimaki (2006) for a survey of the economics literature on bandit problems). In our setting, 
the buyer experiments by consuming the product at a price. This experimentation leads her to update her value and hence her payoff. Of course, she always has a 'safe arm' which offers a payoff of 0 , which is the option of breaking the contract.

The model in this paper resembles that of PST. They study revenue maximization in a very general dynamic setting whereas in this paper we study learning in a dynamic quasilinear framework. In fact, they present a special case of our learning model as an application of their results (the assumptions of which we will explicitly discuss as we are defining our model). It should be pointed out however, that they do not derive a closed form solution for either the efficient or the optimal contract even for this special case. They instead make qualitative arguments regarding the optimal and efficient contract and their intuition is corroborated by the closed form solutions of our general model. Lastly, it should be pointed out that this paper bears closest resemblance to Pavan (2007) which is an older working paper version of PST. For completeness, we would like to refer the reader to a few other important papers in the mechanism design literature which are similar in spirit to our work- Baron and Besanko (1984), Battaglini (2005), Courty and Li (2000) and Eso and Szentes (2007), to name but a few.

The paper is organized as follows. Section 2 describes the framework of the model and some key assumptions. Section 3 derives the socially efficient contract. Section 4 sets up the seller's revenue maximization problem and derives its closed form solution. Section 5 presents a simple 2 period example which highlights some of the important features of our results. Section 6 discusses the assumption of independent shocks made in this paper. Finally, section 7 discusses some simple extensions of our model and provides concluding remarks. The appendix contains some of the proofs.

\section{The Model}

The model consists of a single buyer and a single seller. Where not explicitly mentioned the buyer is referred to using feminine pronouns and the seller using masculine pronouns. Contracting occurs over $T$ time periods where individual time periods are denoted by $t \in\{1,2, \ldots, T\}$. We assume that both the buyer and the seller are risk neutral and that the seller has a cost of zero (assuming zero seller cost is purely for ease of exposition as the results in the paper generalize trivially to nonzero seller cost). The discount factor is given by $\delta \in[0,1]$.

The valuation of the buyer at time $t$ is given by $\theta_{t}$. At period 1 , the buyer realizes an initial valuation which is unobserved by the seller. This value is denoted by $\theta_{1} \in \Theta$ where $\Theta$ is the bounded interval $\left[\underline{\theta}_{1}, \bar{\theta}_{1}\right] \subset \mathbb{R}$. $\theta_{1}$ is drawn from a distribution $F$, which is assumed to be strictly increasing on the support $\Theta$. If the buyer consumes the object at a time $t$ she receives a signal from her consumption experience which allows her to revise her valuation. The signal $\xi_{t} \in \Xi_{t} \equiv \Xi \equiv(-\infty, \infty)$ is drawn independently from a distribution $G .{ }^{1}$ We will use $\Xi_{t}$ and $\Xi$ interchangeably for notational

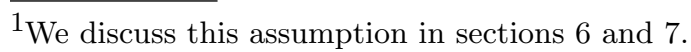


convenience, but the meaning will remain clear. Since we make no assumptions about the support of $G$, this assumption of $\xi_{t}$ being unbounded is without loss of generality. ${ }^{2}$

Let $C^{t}=\left\{c_{1}^{t}, \ldots, c_{\left|C_{t}\right|}^{t}\right\}$ denote the set of time periods up to t where the buyer consumes the object. We denote the set of all possible consumption histories $C^{t}$, at period $t$ by $\mathscr{C}^{t} \equiv 2^{\{1, \ldots, t-1\}}$. For example, if $t=4$ and the buyer consumes the object in periods 1,2 and 4 , then $C^{4}=\left\{c_{1}^{4}, c_{2}^{4}, c_{4}^{4}\right\}=$ $\{1,2,4\}$.

For ease of notation we define the vectors $\xi^{C^{t}}$ and $\xi^{t}$ as follows

$$
\begin{aligned}
\xi^{C^{t}} & =\left(\xi_{c_{1}^{t}+1}, \ldots, \xi_{c_{\left|C^{t}\right|}+1}\right) \\
\xi^{t} & =\left(\xi_{2}, \ldots, \xi_{t}\right)
\end{aligned}
$$

$\xi^{C^{t}}$ denotes the vector of signals the buyer receives up to period $t+1$ due to consumption history $C^{t}$. In our example when $C^{4}=\{1,2,4\}, \xi^{C^{4}}=\left(\xi_{2}, \xi_{3}, \xi_{5}\right)$.

Values evolve according to valuation functions $v$, which depend on the initial valuation $\theta_{1}$ and subsequent signals $\xi_{t}$. Hence if the buyer receives $r-1$ signals $v_{r}$ is defined as $v_{r}: \Theta \times \Xi^{r-1} \rightarrow \mathbb{R}$. We assume these value functions are Markov, that is, for any $\theta_{1}, \xi^{r}$ and $\tilde{\theta}_{1}, \tilde{\xi}^{r}$ such that $v_{r}\left(\theta_{1}, \xi^{r}\right)=$ $v_{r}\left(\tilde{\theta}_{1}, \tilde{\xi}^{r}\right)$, it must be the case that for all $s>r$

$$
v_{s}\left(\theta_{1}, \xi^{r}, \xi_{r+1}, \ldots, \xi_{s}\right)=v_{s}\left(\tilde{\theta}_{1}, \tilde{\xi}^{r}, \xi_{r+1}, \ldots, \xi_{s}\right)
$$

We can now define period $t$ valuation $\theta_{t}$ in terms of the initial value and the subsequent consumption history $C^{t-1}$ (with $r=\left|C^{t-1}\right|$ ) by using valuation functions $v$.

$$
\theta_{t}=v_{t}^{C^{t-1}}\left(\theta_{1}, \xi^{C^{t-1}}\right)=v_{r}\left(\theta_{1}, \xi^{C^{t-1}}\right)
$$

This function defines how the buyer's valuation evolves as she receives new signals from her experimentation. It is assumed that the seller knows the functions $v$ and the distributions $F$ and $G$. We will make the following key assumptions about the distribution of initial value $F$ and value functions $v$.

A1 The density $f$ of cdf $F$ is log concave, implying the monotone hazard rate condition.

A2 $v_{r}$ is differentiable and strictly increasing in all arguments.

A3 For $2 \leq r \leq s, \lim _{\xi_{r} \rightarrow \infty} v_{s}\left(\theta_{1}, \xi^{s}\right) \rightarrow \infty$ and $\lim _{\xi_{r} \rightarrow-\infty} v_{s}\left(\theta_{1}, \xi^{s}\right) \rightarrow-\infty$.

A4 $v_{s}\left(\theta_{1}, \xi^{s}\right)$ are concave in $\theta_{1}$ and $\frac{\partial^{2} v_{s}\left(\theta_{1}, \xi^{s}\right)}{\partial \theta_{1} \partial \xi_{r}} \leq 0$ for any $\left(\theta_{1}, \xi^{s}\right), r \leq s$.

A5 For any $r \geq 3$, any $s \geq r$ and any $\left(\theta_{1}, \xi^{r}, \xi_{r+1}, \ldots, \xi_{s}\right)$

$$
\frac{\partial^{2} v_{s}\left(\theta_{1}, \xi^{r}, \xi_{r+1}, \ldots, \xi_{s}\right)}{\partial \theta_{1} \partial \xi_{r-1}} \frac{\partial v_{r}\left(\theta_{1}, \xi^{r}\right)}{\partial \xi_{r}} \leq \frac{\partial^{2} v_{s}\left(\theta_{1}, \xi^{r}, \xi_{r+1}, \ldots, \xi_{s}\right)}{\partial \theta_{1} \partial \xi_{r}} \frac{\partial v_{r}\left(\theta_{1}, \xi^{r}\right)}{\partial \xi_{r-1}}
$$

Similarly for $r=2$ and any $\left(\theta_{1}, \xi_{2}, \ldots \xi_{s}\right), s \geq 2$

$$
\frac{\partial^{2} v_{s}\left(\theta_{1}, \xi_{2}, \ldots, \xi_{s}\right)}{\partial \theta_{1}^{2}} \frac{\partial v_{2}\left(\theta_{1}, \xi_{2}\right)}{\partial \xi_{2}} \leq \frac{\partial^{2} v_{s}\left(\theta_{1}, \xi_{2}, \ldots, \xi_{s}\right)}{\partial \theta_{1} \partial \xi_{2}} \frac{\partial v_{2}\left(\theta_{1}, \xi_{2}\right)}{\partial \theta_{1}}
$$

${ }^{2}$ The assumption implies that the mechanism must be defined for reported values that are potentially outside the support of $G$. However, in equilibrium the only values that the buyer will report will lie in the support. 
These assumptions are identical to those required by Pavan (2007). Assumption A1 is the standard monotone hazard rate assumption used in much of the mechanism design literature. Assumptions $\mathrm{A} 2$ and A3 are natural properties of a value function and are hence innocuous. Assumption A4 is an intuitive concavity condition, which one would expect a dynamic valuation function to satisfy. Assumption A5, admittedly has no obvious economic interpretation. It is purely a technical assumption required for our results. As a justification, we observe that a number of natural value functions satisfy our assumptions. Consider for example the following value functions.

$$
\begin{aligned}
& \theta_{t}=v_{t}\left(\theta_{1}, \xi^{t}\right)=\theta_{1}+\sum_{i=1}^{t} \xi_{t} \\
& \theta_{t}=v_{t}\left(\theta_{1}, \xi^{t}\right)=\alpha_{1}^{t} \theta_{t-1}+\cdots+\alpha_{k}^{t} \theta_{t-k}+\xi_{t} \\
& \theta_{t}=v_{t}\left(\theta_{1}, \xi^{t}\right)=\theta_{1} \times \prod_{i=1}^{t} \xi_{t}
\end{aligned}
$$

The value function (1) is the random walk case and is a special case of a general linear autoregressive process of order $k(\operatorname{AR}(k))$, in which values are defined recursively by equation (2). Finally, (3) is an example of a value function where the shocks are multiplicative.

We now define the message space. At period 1 , the set of messages is denoted by $M_{1}$. At any period $t>1$, if the buyer receives the object at period $t-1$, she receives a signal $\xi_{t}$ about her valuation from her consumption experience and she can report a message from message space $M_{t}$. If she does not receive the object at $t-1$ then she has no new private information and hence does not make a report. The seller commits to a mechanism consisting of a series of functions $q$, $p$, which depend on the current time period $t$, the history of buyer consumption $C^{t-1}$ and the history of reported messages. $q$ denotes the probability of receiving the object and $p$ is the price. More formally

$$
\begin{aligned}
& q_{t}^{C^{t-1}}: M_{1} \times M_{c_{1}^{t-1}+1} \times \cdots \times M_{c_{r}^{t-1}+1} \rightarrow[0,1] \\
& p_{t}^{C^{t-1}}: M_{1} \times M_{c_{1}^{t-1}+1} \times \cdots \times M_{c_{r}^{t-1}+1} \rightarrow \mathbb{R} \\
& \text { where } r=\left|C^{t-1}\right|
\end{aligned}
$$

Returning to the above 5 period example, where $C^{4}=\{1,2,4\}$. At period 5 , the mechanism offers a contract $\left(q_{5}^{C^{4}}, p_{5}^{C^{4}}\right)$ where $q_{5}^{C^{4}}: M_{1} \times M_{2} \times M_{3} \times M_{5} \rightarrow[0,1]$ and similarly $p_{5}^{C^{4}}: M_{1} \times M_{2} \times M_{3} \times M_{5} \rightarrow$ $\mathbb{R}$. In other words since the buyer does not receive the object in period 3 , she has no new private information, and hence does not report in period 4. However, she ends up consuming the object in period 4 and therefore once again makes a report in period 5 .

Note that while we force the seller to commit to a contract at period 1, we allow the buyer to walk away and end the contract at any time period. Because the contract cannot be renegotiated, this does not give the buyer additional bargaining power by threatening to walk away. It merely forces the contract to be individually rational for the buyer at all time periods. The timing and the contracting are made more explicit below.

- The buyer learns her initial value $\theta_{1}$. 
- The seller offers a mechanism given by $\left(q_{1}, p_{1}\right),\left(q_{2}^{C^{1}}, p_{2}^{C^{1}}\right), \ldots,\left(q_{T}^{C^{T-1}}, p_{T}^{C^{T-1}}\right)$ for all $C^{1} \in$ $\mathscr{C}^{1}, \ldots, C^{T-1} \in \mathscr{C}^{T-1}$.

- The buyer reports a message $m_{1} \in M_{1}$.

- Having reported $m_{1}$, she is offered the object with probability $q_{1}\left(m_{1}\right)$ with corresponding transfer $p_{1}\left(m_{1}\right)$. If she agrees then the contract is enacted, otherwise, if she refuses to participate then both she and the seller get a payoff of 0 and the contract terminates.

- If she receives the object (if $q_{1}\left(m_{1}\right)<1$, she might not), she receives signal $\xi_{2}$ through her consumption experience. If she doesn't receive the object, she gets no further information.

- If she ends up receiving the object in period 1 , she reports a message $m_{2} \in M_{2}$ and is offered $q_{2}^{C^{1}}\left(m_{1}, m_{2}\right), p_{2}^{C^{1}}\left(m_{1}, m_{2}\right)$ where $C^{1}=\{1\}$. If she agrees then the contract is enacted, otherwise, if she refuses to participate then both she and the seller get a payoff of 0 in period 2 and the contract terminates.

- If she does not end up receiving the object in period 1 , she is offered $q_{2}^{C^{1}}\left(m_{1}\right), p_{2}^{C^{1}}\left(m_{1}\right)$ where $C^{1}=\phi$. If she agrees then the contract is enacted, otherwise, if she refuses to participate then both she and the seller get a payoff of 0 in period 2 and the contract terminates.

- This process continues for the duration $T$ or until the buyer decides to break the contact.

Finally, we observe that since the seller commits to the full $T$ period mechanism, the Revelation Principle applies and we need only consider direct mechanisms. In other words, we need only consider mechanisms where $M_{1} \equiv \Theta$ and $M_{t} \equiv \Xi_{t}$. In all subsequent sections we analyze only the direct mechanisms

$$
\begin{aligned}
& q_{t}^{C^{t-1}}: \Theta \times \Xi_{c_{1}^{t-1}+1} \times \cdots \times \Xi_{c_{r}^{t-1}+1} \rightarrow[0,1] \\
& p_{t}^{C^{t-1}}: \Theta \times \Xi_{c_{1}^{t-1}+1} \times \cdots \times \Xi_{c_{r}^{t-1}+1} \rightarrow \mathbb{R} \\
& \text { where } r=\left|C^{t-1}\right|
\end{aligned}
$$

PST study a special case of this learning model. They assume that the initial valuation and subsequent shocks are normally distributed. In their setting, the $t$ period valuation $\theta_{t}$ is a weighted average of value $\theta_{t-1}$ and signal $\xi_{t}$. Clearly this is a special case of our setting. Moreover, they also assume that the seller offers a contract $q_{t}$ at each period where $q_{t} \in\{0,1\}$. In other words they do not allow the seller to offer probabilistic contracts to the buyer. Lastly, they do not derive closed form solutions for the efficient and seller optimal contract which is in contrast to this paper.

\section{The Efficient Policy}

We start off by deriving the efficient policy. Since the seller has no cost, the ex-ante efficient policy maximizes the following surplus function

$$
\theta_{1} q_{1}\left(\theta_{1}\right)+\sum_{t=2}^{T} \sum_{C^{t-1} \in \mathscr{C}^{t-1}} \delta^{t} \int_{\Xi_{2}} \ldots \int_{\Xi_{T}} \mu_{q}\left(C^{t-1}\right) q_{t}^{C^{t-1}}\left(\theta_{1}, \xi^{C^{t-1}}\right) v_{t}^{C^{t-1}}\left(\theta_{1}, \xi^{C^{t-1}}\right) d G\left(\xi_{2}\right) \ldots d G\left(\xi_{T}\right)
$$


Where $\mu_{q}\left(C^{t-1}\right)$ is the probability that history of consumption $C^{t-1}$ occurs as a result of contract q. Hence,

$$
\mu_{q}\left(C^{t-1}\right)=\prod_{s=2}^{t-1} w_{s}^{D^{s-1}}\left(\theta_{1}, \xi^{D^{s-1}}\right)
$$

where $D^{s-1}=C^{t-1} \cap\{1, \ldots, s-1\}$

and

$$
w_{s}^{D^{s-1}}\left(\theta_{1}, \xi^{D^{s-1}}\right)= \begin{cases}q_{s}^{D^{s-1}}\left(\theta_{1}, \xi^{D^{s-1}}\right) & \text { if } s \in C^{t-1} \\ 1-q_{s}^{D^{s-1}}\left(\theta_{1}, \xi^{D^{s-1}}\right) & \text { if } s \notin C^{t-1}\end{cases}
$$

We now derive the efficient contract by backward induction. At time period $T$, for any history of consumption $C^{T-1}$, the efficient contract must be

$$
q_{T}^{C^{T-1}}\left(\theta_{1}, \xi^{C^{T-1}}\right)= \begin{cases}1 & \text { if } v_{T}^{C^{T-1}}\left(\theta_{1}, \xi^{C^{T-1}}\right) \geq 0 \\ 0 & \text { otherwise }\end{cases}
$$

Similarly, at time period $T-1$, for any history of consumption $C^{T-2}$, the efficient contract will be

$$
q_{T-1}^{C^{T-2}}\left(\theta_{1}, \xi^{C^{T-2}}\right)= \begin{cases}1 & \text { if } v_{T-1}^{C^{T-2}}\left(\theta_{1}, \xi^{C^{T-2}}\right)+ \\ & \delta \int_{\Xi_{T}} q_{T}^{C^{T-1}}\left(\theta_{1}, \xi^{C^{T-1}}, \xi_{T}\right) v_{T}^{C^{T-1}}\left(\theta_{1}, \xi^{C^{T-2}}, \xi_{T}\right) d G\left(\xi_{T}\right) \geq 0 \\ 0 & \text { otherwise }\end{cases}
$$

where $C^{T-1}=C^{T-2} \cup\{T-1\}$ and $q_{T}$ is defined above. We observe that it is never efficient to not allocate the object in period $T-1$ and then allocate it in period $T$. This is because, from the definition of $q_{T}$, the second term $\delta \int_{\Xi_{T}} q_{T}^{C^{T-1}}\left(\theta_{1}, \xi^{C^{T-1}}, \xi_{T}\right) v_{T}^{C^{T-1}}\left(\theta_{1}, \xi^{C^{T-2}}, \xi_{T}\right) d G\left(\xi_{T}\right)$ is always non-negative. Hence, if the object is not allocated in period $T-1$, it must be the case that $v_{T-1}^{C^{T-2}}\left(\theta_{1}, \xi^{C^{T-2}}\right)<0$ and hence the object will not be allocated in period $T$ either. Conversely, notice that the efficient policy could allocate the object to the consumer even though her period $T-1$ flow valuation $v_{T-1}^{C^{T-2}}\left(\theta_{1}, \xi^{C^{T-2}}\right)$ is negative. This is because there could be positive welfare gains from experimentation.

Lastly, we also observe that $q^{T-1}$ and $q^{T}$ never take a value between 0 and 1 . When $v_{T}^{C^{T-1}}\left(\theta_{1}, \xi^{C^{T-1}}\right)=$ $0, q_{T}^{C^{T-1}}$ could potentially take a value between 0 and 1 but this will be outcome equivalent to $q_{T}^{C^{T-1}}=1$. The same argument can be applied to $q_{T-1}^{C^{T-2}}$.

This argument can be backward inducted to period 1 . We denote histories $S^{t}=\{1, \ldots, t-1\}$. These are histories at time $t$ where the buyer gets the object in every period up to $t$. Hence, at these histories the value function $v_{t}^{S^{t}}$ is simply equal to $v_{t}$.

Proposition 1. The efficient contract is given by the following quantities.

$$
q_{T}^{C^{T}}\left(\theta_{1}, \xi^{C^{T}}\right)= \begin{cases}1 & \text { if } C^{T}=S^{T}=\{1, \ldots, T-1\} \text { and } \\ & v_{T}\left(\theta_{1}, \xi^{S^{T}}\right) \geq 0 \\ 0 \quad & \text { otherwise }\end{cases}
$$


$q_{t}^{C^{t}}\left(\theta_{1}, \xi^{C^{t}}\right)= \begin{cases}1 & \text { if } C^{t}=S^{t}=\{1, \ldots, t-1\} \text { and } \\ & v_{t}\left(\theta_{1}, \xi^{S^{t}}\right)+ \\ & \sum_{s=t+1}^{T} \int_{\Xi_{t+1}} \ldots \int_{\Xi_{T}} \delta^{s-t}\left(\prod_{r=t+1}^{s} q_{r}^{S^{r}}\left(\theta_{1}, \xi^{S^{r}}\right)\right) v_{s}\left(\theta_{1}, \xi^{S^{s}}\right) d G\left(\xi_{t+1}\right) \ldots d G\left(\xi_{T}\right) \geq 0 \\ 0 & \text { otherwise }\end{cases}$

The efficient contract will allocate the object to the buyer with probability 1 as long as the sum of their value and their expected value from experimentation is non-negative. The efficient contract induces efficient experimentation, that is, if the flow value $v_{t}$ of the buyer falls below 0 , she is allowed to experiment if her value is expected to improve with experimentation. Also the efficient contract is a stopping rule where if the buyer does not receive the object in period $t$ she will not receive it in any subsequent period.

\section{The Seller Optimal Mechanism}

In this section we will derive the seller optimal contract for 2 periods. This will highlight the key steps of the proof without the reader having to deal with complicated notation. We will state the result for the general $T$ period case at the end of the section and provide the proof of incentive compatibility in the appendix.

We start off by defining the buyer's utility at period 2. There are two possible histories which we denote by $C_{1}^{2}=\{1\}$ and $C_{2}^{2}=\phi$. When the buyer receives the object in period 1 having reported $\hat{\theta}_{1}$ (with true value $\theta_{1}$ ), she receives a signal given by $\xi_{2}$. Her utility in period 2 is then given in terms of her true valuations $\theta_{1}, \xi_{2}$, reported valuations $\hat{\theta}_{1}, \hat{\xi}_{2}$ and history of consumption $C_{1}^{2}$ as follows

$$
\tilde{U}_{2}^{C_{1}^{2}}\left(\theta_{1}, \xi_{2} ; \hat{\theta}_{1}, \hat{\xi}_{2}\right)=v_{2}\left(\theta_{1}, \xi_{2}\right) q_{2}^{C_{1}^{2}}\left(\hat{\theta}_{1}, \hat{\xi}_{2}\right)-p_{2}^{C_{1}^{2}}\left(\hat{\theta}_{1}, \hat{\xi}_{2}\right)
$$

The tilde superscript is used for utility functions where the buyer has new information to report. The semicolon is used to separate the true from the reported values. If she does not receive the object her utility at period 2 is simply given by

$$
\hat{U}_{2}^{C_{2}^{2}}\left(\theta_{1} ; \hat{\theta}_{1}\right)=\theta_{1} q_{2}^{C_{2}^{2}}\left(\hat{\theta}_{1}\right)-p_{2}^{C_{2}^{2}}\left(\hat{\theta}_{1}\right)
$$

If the buyer received the object at $t=1$ by reporting value $\hat{\theta}_{1}$ and has realized true value and signal $\theta_{1}, \xi_{2}$, the highest utility she can get at $t=2$ is given by the solution to the following optimization problem.

$$
\hat{U}_{2}^{C_{1}^{2}}\left(\theta_{1}, \xi_{2} ; \hat{\theta}_{1}\right)=\max _{\hat{\xi}_{2}}\left\{v_{2}\left(\theta_{1}, \xi_{2}\right) q_{2}^{C_{1}^{2}}\left(\hat{\theta}_{1}, \hat{\xi}_{2}\right)-p_{2}^{C_{1}^{2}}\left(\hat{\theta}_{1}, \hat{\xi}_{2}\right)\right\}
$$

The hat superscript on utility functions is used denote the maximum obtainable utility for the buyer, contingent on both the true values of the signals and the previously reported values of the signals. In other words

$$
\hat{U}_{2}^{C_{1}^{2}}\left(\theta_{1}, \xi_{2}, ; \hat{\theta}_{1}\right)=\max _{\hat{\xi}_{2}} \tilde{U}_{2}^{C_{1}^{2}}\left(\theta_{1}, \xi_{2} ; \hat{\theta}_{1}, \hat{\xi}_{2}\right)
$$

We now define incentive compatibility in this context. 
Definition 4.1 (Incentive Compatibility). A mechanism $(q, p)$ is incentive compatible if truthtelling is optimal for the buyer at period 1 and truthtelling is optimal for all other periods $t$ (where she has new private information to report) conditional on having reported truthfully in the past.

This is clearly a weaker requirement than requiring truthtelling to be optimal regardless of past reports. The seller optimal contract will turn out to be the same under either requirement. Formally, if the buyer reveals her value truthfully at $t=1$ and receives the object then truth telling is incentive compatible at $t=2$ if

$$
\tilde{U}_{2}^{C_{1}^{2}}(\theta, \xi ; \theta, \xi)=\hat{U}_{2}^{C_{1}^{2}}(\theta, \xi ; \theta)=U_{2}^{C_{1}^{2}}(\theta, \xi)
$$

where $U_{2}(\theta, \xi)$ is concise notation for utility from truth telling. Clearly, when she does not receive the object she has no new private information and hence there are no incentive issues at period 2 . We define the expected utility of the buyer having realized value $\theta$ at $t=1$ and reporting optimally as follows

(6) $\hat{U}_{1}\left(\theta_{1}\right)=\max _{\hat{\theta}_{1}}\left\{\theta_{1} q_{1}\left(\hat{\theta}_{1}\right)-p_{1}\left(\hat{\theta}_{1}\right)+\delta q_{1}\left(\hat{\theta}_{1}\right) \int_{\Xi} \hat{U}_{2}^{C_{1}^{2}}\left(\theta_{1}, \xi_{2} ; \hat{\theta}_{1}\right) d G\left(\xi_{2}\right)+\delta\left(1-q_{1}\left(\hat{\theta}_{1}\right)\right) \hat{U}_{2}^{C_{2}^{2}}\left(\theta_{1} ; \hat{\theta}_{1}\right)\right\}$

Now, we can define the utility from truth telling in both periods as follows.

$$
U_{1}\left(\theta_{1}\right)=\theta_{1} q_{1}\left(\theta_{1}\right)-p_{1}\left(\theta_{1}\right)+\delta q_{1}\left(\theta_{1}\right) \int_{\Xi} U_{2}^{C_{1}^{2}}\left(\theta_{1}, \xi_{2}\right) d G\left(\xi_{2}\right)+\delta\left(1-q_{1}\left(\theta_{1}\right)\right) U_{2}^{C_{2}^{2}}\left(\theta_{1}\right)
$$

The revenue of the seller when the buyer reports truthfully in both periods is given by

$$
\int_{\Theta}\left[p_{1}\left(\theta_{1}\right)+\delta q_{1}\left(\theta_{1}\right) \int_{\Xi} p_{2}^{C_{1}^{2}}\left(\theta_{1}, \xi_{2}\right) d G\left(\xi_{2}\right)+\delta\left(1-q_{1}\left(\theta_{1}\right)\right) p_{2}^{C_{2}^{2}}\left(\theta_{1}\right)\right] d F\left(\theta_{1}\right)
$$

A mechanism is said to be incentive compatible if truth telling maximizes expected utility for the buyer in period 1 and truth telling is also optimal in period 2, conditional on having reported truthfully in period 1. In other words, $U_{1}\left(\theta_{1}\right)=\hat{U}_{1}\left(\theta_{1}\right)$ and (5) should hold simultaneously. Individual rationality requires that $U_{1}\left(\theta_{1}\right) \geq 0, U_{2}^{C_{1}^{2}}\left(\theta_{1}, \xi_{2}\right) \geq 0$ and $U_{2}^{C_{2}^{2}}\left(\theta_{1}\right) \geq 0$ for all $\left(\theta_{1}, \xi_{2}\right) \in \Theta \times \Xi_{2}$.

The seller designs an optimal contract by solving the following problem.

$$
\begin{aligned}
\max _{q_{1}(\cdot), p_{1}(\cdot), q_{2}(\cdot, \cdot), p_{2}(\cdot, \cdot)} \int_{\Theta}\left[p_{1}\left(\theta_{1}\right)+\delta q_{1}\left(\theta_{1}\right)\right. & \left.\int_{\Xi_{2}} p_{2}^{C_{1}^{2}}\left(\theta_{1}, \xi_{2}\right) d G\left(\xi_{2}\right)+\delta\left(1-q_{1}\left(\theta_{1}\right)\right) p_{2}^{C_{2}^{2}}\left(\theta_{1}\right)\right] d F\left(\theta_{1}\right) \\
\text { subject to } \quad U_{1}\left(\theta_{1}\right) & =\hat{U}_{1}\left(\theta_{1}\right), \quad U_{2}\left(\theta_{1}, \xi_{2}\right)=\hat{U}_{2}\left(\theta_{1}, \xi_{2} ; \theta_{1}\right) \\
U_{1}\left(\theta_{1}\right) & \geq 0, U_{2}^{C_{1}^{2}}\left(\theta_{1}, \xi_{2}\right) \geq 0, U_{2}^{C_{2}^{2}}\left(\theta_{1}\right) \geq 0
\end{aligned}
$$

In order to solve this problem we use a similar reverse engineering process as the one in Eso and Szentes (2007). We first derive necessary conditions for incentive compatibility and define a relaxed optimization problem in which the seller is maximizing his revenue subject to these necessary conditions.

We start by examining the incentive constraint in period 2. Using the standard technique of Myerson (1981), equation (4) implies that a necessary and sufficient condition for truth telling at 
$t=2$ given truthful reporting at $t=1$ is

$$
U_{2}^{C_{1}^{2}}\left(\theta_{1}, \xi_{2}\right)=U_{2}^{C_{1}^{2}}\left(\theta_{1}, \underline{\xi}_{2}\right)+\int_{-\infty}^{\xi_{2}} \frac{\partial v\left(\theta_{1}, \tilde{\xi}_{2}\right)}{\partial \tilde{\xi}_{2}} q_{2}^{C_{1}^{2}}\left(\theta_{1}, \tilde{\xi}_{2}\right) d \tilde{\xi}_{2}
$$

where $U_{2}^{C_{1}^{2}}\left(\theta_{1}, \underline{\xi}_{2}\right)=\lim _{\xi_{2} \rightarrow-\infty} U_{2}^{C_{1}^{2}}\left(\theta_{1}, \xi_{2}\right)$. We can use the envelope theorem to differentiate ${ }^{3}$ equation (6) with respect to $\theta_{1}$ to get

$$
\frac{d U_{1}\left(\theta_{1}\right)}{d \theta_{1}}=q_{1}\left(\tilde{\theta}_{1}\right)+\delta q_{1}\left(\tilde{\theta}_{1}\right) \int_{\Xi_{2}} \frac{\partial \hat{U}_{2}^{C_{1}^{2}}\left(\theta_{1}, \xi_{2} ; \tilde{\theta}_{1}\right)}{\partial \theta_{1}} d G\left(\xi_{2}\right)+\delta\left(1-q_{1}\left(\theta_{1}\right)\right) \frac{\partial \hat{U}_{2}^{C_{2}^{2}}\left(\theta_{1} ; \tilde{\theta}_{1}\right)}{\partial \theta_{1}}
$$

where $\tilde{\theta}_{1}$ is assumed to be the maximizer of (6). We can use the envelope theorem again on (4) yielding

$$
\frac{\partial \hat{U}_{2}^{C_{1}^{2}}\left(\theta_{1}, \xi_{2} ; \tilde{\theta}_{1}\right)}{\partial \theta_{1}}=\frac{\partial v_{2}\left(\theta_{1}, \xi_{2}\right)}{\partial \theta_{1}} q_{2}^{C_{1}^{2}}\left(\tilde{\theta}_{1}, \tilde{\xi}_{2}\right)
$$

where $\tilde{\xi}_{2}$ is assumed to be the maximizer of (4). We can do the same for $\hat{U}_{2}^{C_{2}^{2}}$. Plugging these into equation (8) we arrive at a necessary condition for truth telling:

$$
\frac{d U_{1}\left(\theta_{1}\right)}{d \theta_{1}}=q_{1}\left(\theta_{1}\right)+\delta q_{1}\left(\theta_{1}\right) \int_{\Xi_{2}} \frac{\partial v_{2}\left(\theta_{1}, \xi_{2}\right)}{\partial \theta_{1}} q_{2}^{C_{1}^{2}}\left(\theta_{1}, \xi_{2}\right) d G\left(\xi_{2}\right)+\delta\left(1-q_{1}\left(\theta_{1}\right)\right) q_{2}^{C_{2}^{2}}\left(\theta_{1}\right)
$$

While this condition is necessary, it is not, in general, sufficient for truth telling to be incentive compatible. This is in contrast with the static model and this observation has also been made by Eso and Szentes (2007) and Pavan (2007). (9) integrates to

$$
(\mathrm{ENV} 1) U_{1}\left(\theta_{1}\right)=U_{1}\left(\underline{\theta}_{1}\right)+\int_{\underline{\theta}_{1}}^{\theta_{1}}\left[q_{1}(t)+\delta q_{1}(t) \int_{\Xi_{2}} \frac{\partial v\left(t, \xi_{2}\right)}{\partial t} q_{2}^{C_{1}^{2}}\left(t, \xi_{2}\right) d G\left(\xi_{2}\right)+\delta\left(1-q_{1}(t)\right) q_{2}^{C_{2}^{2}}(t)\right] d t
$$

We can now rewrite revenue equation (7) in terms of the buyer's utility and define the seller's revenue as

$$
\int_{\Theta}\left[\theta_{1} q_{1}\left(\theta_{1}\right)+\delta q_{1}\left(\theta_{1}\right) \int_{\Xi_{2}} v\left(\theta_{1}, \xi_{2}\right) q_{2}^{C_{1}^{2}}\left(\theta_{1}, \xi_{2}\right) d G\left(\xi_{2}\right)+\delta\left(1-q_{1}\left(\theta_{1}\right)\right) \theta_{1} q_{2}^{C_{2}^{2}}\left(\theta_{1}\right)-U_{1}\left(\theta_{1}\right)\right] d F\left(\theta_{1}\right)
$$

We now define the relaxed problem for the seller. In this problem he maximizes his revenue subject to the necessary conditions for incentive compatibility that we have derived:

$$
\begin{aligned}
\max _{q_{1}(\cdot), U_{1}(\cdot), q_{2}(\cdot, \cdot), U_{2}^{C}(\cdot, \cdot)} \int_{\Theta}[ & \theta_{1} q_{1}\left(\theta_{1}\right)+\delta q_{1}\left(\theta_{1}\right) \int_{\Xi_{2}} v_{2}\left(\theta_{1}, \xi_{2}\right) q_{2}^{C_{1}^{2}}\left(\theta_{1}, \xi_{2}\right) d G\left(\xi_{2}\right)+\delta\left(1-q_{1}\left(\theta_{1}\right)\right) \theta q_{2}^{C_{2}^{2}}\left(\theta_{1}\right) \\
& \left.-U_{1}\left(\theta_{1}\right)\right] d F\left(\theta_{1}\right)
\end{aligned}
$$

subject to $\quad(\mathrm{ENV} 1), \quad(\mathrm{ENV} 2), \quad U_{1}\left(\theta_{1}\right) \geq 0, \quad U_{2}^{C_{1}^{2}}\left(\theta_{1}, \xi_{2}\right) \geq 0, \quad U_{2}^{C_{2}^{2}}\left(\theta_{1}\right) \geq 0$

\footnotetext{
${ }^{3}$ We derive this condition explicitly in the appendix as there is no immediately obvious reason to assume that the buyer's utility is differentiable. This derivation will also show why the envelope condition is necessary but not sufficient for incentive compatibility.
} 
By substituting equation (ENV1) into the objective function of the above relaxed problem and integrating by parts, we can rewrite the objective function as

$$
\begin{aligned}
\int_{\Theta}[\quad & \left(\theta_{1}-\frac{1-F\left(\theta_{1}\right)}{f\left(\theta_{1}\right)}\right) q_{1}\left(\theta_{1}\right)+ \\
& \delta q_{1}\left(\theta_{1}\right) \int_{\Xi_{2}}\left(v_{2}\left(\theta_{1}, \xi_{2}\right)-\frac{\partial v_{2}\left(\theta_{1}, \xi_{2}\right)}{\partial \theta_{1}} \frac{1-F\left(\theta_{1}\right)}{f\left(\theta_{1}\right)}\right) q_{2}^{C_{1}^{2}}\left(\theta_{1}, \xi_{2}\right) d G\left(\xi_{2}\right)+ \\
& \left.\delta\left(1-q_{1}\left(\theta_{1}\right)\right)\left(\theta_{1}-\frac{1-F\left(\theta_{1}\right)}{f\left(\theta_{1}\right)}\right) q_{2}^{C_{2}^{2}}\left(\theta_{1}\right)-U_{1}\left(\underline{\theta_{1}}\right)\right] d F\left(\theta_{1}\right)
\end{aligned}
$$

It is then immediate that the solution to the relaxed problem is independent of participation and incentive constraints for period 2. These constraints are only relevant for deriving the prices. The following solution of the relaxed problem then follows

Proposition 2. The solution to the two period seller's relaxed problem is given by $U_{1}\left(\underline{\theta_{1}}\right)=0$ and the following allocation rules:

$$
\begin{aligned}
q_{2}^{C_{1}^{2}}\left(\theta_{1}, \xi_{2}\right) & = \begin{cases}1 & \text { if } v_{2}\left(\theta_{1}, \xi_{2}\right)-\frac{1-F\left(\theta_{1}\right)}{f\left(\theta_{1}\right)} \frac{\partial v_{2}\left(\theta_{1}, \xi_{2}\right)}{\partial \theta_{1}} \geq 0 \\
0 & \text { otherwise }\end{cases} \\
q_{2}^{C_{2}^{2}}\left(\theta_{1}\right) & =0 \\
q_{1}\left(\theta_{1}\right) & = \begin{cases}1 & \text { if }\left[\theta_{1}-\frac{1-F\left(\theta_{1}\right)}{f\left(\theta_{1}\right)}\right]+\delta \int_{\Xi}\left[v_{2}\left(\theta_{1}, \xi_{2}\right)-\frac{1-F\left(\theta_{1}\right)}{f\left(\theta_{1}\right)} \frac{\partial v_{2}\left(\theta_{1}, \xi_{2}\right)}{\partial \theta_{1}}\right] q_{2}^{C_{1}^{2}}\left(\theta_{1}, \xi_{2}\right) d G\left(\xi_{2}\right) \geq 0 \\
0 & \text { otherwise }\end{cases}
\end{aligned}
$$

These quantities are derived by pointwise maximization of the seller revenue function (10). This can be seen as follows. The second term of (10) is clearly maximized when $q_{2}^{C_{1}^{2}}\left(\theta_{1}, \xi_{2}\right)$ is given as above. The third term is maximized when

$$
q_{2}^{C_{2}^{2}}\left(\theta_{1}\right)= \begin{cases}1 & \text { if } \theta_{1}-\frac{1-F\left(\theta_{1}\right)}{f\left(\theta_{1}\right)} \geq 0 \\ 0 & \text { otherwise }\end{cases}
$$

But then the optimal selection of $q_{1}$ when will be given by

$$
q_{1}\left(\theta_{1}\right)=\left\{\begin{aligned}
1 \quad & \text { if }\left[\theta_{1}-\frac{1-F\left(\theta_{1}\right)}{f\left(\theta_{1}\right)}\right]+\delta \int_{\Xi_{2}}\left[v_{2}\left(\theta_{1}, \xi_{2}\right)-\frac{1-F\left(\theta_{1}\right)}{f\left(\theta_{1}\right)} \frac{\partial v_{2}\left(\theta_{1}, \xi_{2}\right)}{\partial \theta_{1}}\right] q_{2}^{C_{1}^{2}}\left(\theta_{1}, \xi_{2}\right) d G\left(\xi_{2}\right) \\
& \geq \delta\left[\theta_{1}-\frac{1-F\left(\theta_{1}\right)}{f\left(\theta_{1}\right)}\right] q_{2}^{C_{2}^{2}}\left(\theta_{1}\right) \\
0 & \text { otherwise }
\end{aligned}\right.
$$

But from the derived value of $q_{2}^{C_{1}^{2}}$, it is always the case that

$$
\left[\theta_{1}-\frac{1-F\left(\theta_{1}\right)}{f\left(\theta_{1}\right)}\right]+\delta \int_{\Xi_{2}}\left[v_{2}\left(\theta_{1}, \xi_{2}\right)-\frac{1-F\left(\theta_{1}\right)}{f\left(\theta_{1}\right)} \frac{\partial v_{2}\left(\theta_{1}, \xi_{2}\right)}{\partial \theta_{1}}\right] q_{2}^{C_{1}^{2}}\left(\theta_{1}, \xi_{2}\right) d G\left(\xi_{2}\right) \delta \geq\left[\theta_{1}-\frac{1-F\left(\theta_{1}\right)}{f\left(\theta_{1}\right)}\right]
$$


as the second term on the left hand side is always non-negative. Moreover, since $0 \leq q_{2}^{C_{2}^{2}} \leq 1$, it is always the case that

$$
\begin{aligned}
& {\left[\theta_{1}-\frac{1-F\left(\theta_{1}\right)}{f\left(\theta_{1}\right)}\right]+\delta \int_{\Xi_{2}}\left[v_{2}\left(\theta_{1}, \xi_{2}\right)-\frac{1-F\left(\theta_{1}\right)}{f\left(\theta_{1}\right)} \frac{\partial v_{2}\left(\theta_{1}, \xi_{2}\right)}{\partial \theta_{1}}\right] q_{2}^{C_{1}^{2}}\left(\theta_{1}, \xi_{2}\right) d G\left(\xi_{2}\right) \geq 0 } \\
\Longrightarrow \quad & {\left[\theta_{1}-\frac{1-F\left(\theta_{1}\right)}{f\left(\theta_{1}\right)}\right]+\delta \int_{\Xi_{2}}\left[v_{2}\left(\theta_{1}, \xi_{2}\right)-\frac{1-F\left(\theta_{1}\right)}{f\left(\theta_{1}\right)} \frac{\partial v_{2}\left(\theta_{1}, \xi_{2}\right)}{\partial \theta_{1}}\right] q_{2}^{C_{1}^{2}}\left(\theta_{1}, \xi_{2}\right) d G\left(\xi_{2}\right) } \\
\geq & \delta\left[\theta_{1}-\frac{1-F\left(\theta_{1}\right)}{f\left(\theta_{1}\right)}\right] q_{2}^{C_{2}^{2}}\left(\theta_{1}\right)
\end{aligned}
$$

Hence, whenever $q_{1}\left(\theta_{1}\right)=0$ or history $C_{2}^{2}$ occurs it must be the case that $\theta_{1}-\frac{1-F\left(\theta_{1}\right)}{f\left(\theta_{1}\right)}<0$ or $q_{2}^{C_{2}^{2}}\left(\theta_{1}\right)=0$. Hence, it is outcome equivalent for the seller to define $q_{2}^{C_{2}^{2}}\left(\theta_{1}\right)$ as identically 0 . This will yield the expression for $q_{1}$ in the proposition.

Notice, that the seller optimal contract like the efficient contract, is a stopping rule. If it is not optimal for the seller to allocate the object to the buyer in the first period it will not be optimal to allocate it to her in the second period. Moreover, in the optimal contract $q$ 's do not take values between 0 and 1 . It is straightforward to generalize the intuition of the 2 period result to arbitrary $T$ periods. The $T$ period optimal contract will also be a stopping rule such that if the buyer does not get the object in period $t$, she will not get the object in any period $s>t$. Once again, we denote histories $S^{t}=\{1, \ldots, t-1\}$. Recall, these are histories at time $t$ where the buyer gets the object in every period up to $t$. Hence, at these histories the value function $v_{t}^{S^{t}}$ is simply equal to $v_{t}$.

Proposition 3. The solution to the $T$ period seller's relaxed problem is given by $U_{1}(\underline{\theta})=0$ and the following allocation rules:

$$
\begin{gathered}
q_{T}^{C^{T}}\left(\theta_{1}, \xi^{C^{T}}\right)=\left\{\begin{array}{l}
1 \quad \text { if } C^{T}=S^{T}=\{1, \ldots, T-1\} \text { and } \\
v_{T}\left(\theta_{1}, \xi^{S^{T}}\right)-\frac{1-F\left(\theta_{1}\right)}{f\left(\theta_{1}\right)} \frac{\partial v_{T}\left(\theta_{1}, \xi^{S^{T}}\right)}{\partial \theta_{1}} \geq 0 \\
0 \quad \text { otherwise }
\end{array} q_{t}^{C^{t}}\left(\theta_{1}, \xi^{C^{t}}\right)=\left\{\begin{array}{l}
1 \quad \text { if } C^{t}=S^{t}=\{1, \ldots, t-1\} \text { and } \\
{\left[v_{t}\left(\theta_{1}, \xi^{S^{t}}\right)-\frac{\partial v_{t}\left(\theta_{1}, \xi^{S^{t}}\right)}{\partial \theta_{1}} \frac{1-F\left(\theta_{1}\right)}{f\left(\theta_{1}\right)}\right]+} \\
\sum_{s=t+1}^{T} \int_{\Xi_{t+1}} \ldots \int_{\Xi_{T}} \delta^{s-t}\left(\prod_{r=t+1}^{s} q_{r}^{S^{r}}\left(\theta_{1}, \xi^{S^{r}}\right)\right)\left[v_{s}\left(\theta_{1}, \xi^{S^{s}}\right)-\frac{\partial v_{s}\left(\theta_{1}, \xi^{S^{s}}\right)}{\partial \theta_{1}} \frac{1-F\left(\theta_{1}\right)}{f\left(\theta_{1}\right)}\right] \\
d G\left(\xi_{t+1}\right) \ldots d G\left(\xi_{T}\right) \geq 0 \\
0 \begin{array}{l}
\text { otherwise }
\end{array}
\end{array}\right.\right.
\end{gathered}
$$

The proof of this result uses the identical backward induction procedure of the 2 period case presented in this section and is hence omitted. Interestingly, note that the seller optimal contract is identical to that of the efficient contract with the flow values replaced by the virtual flow values. In the dynamic setting the virtual flow values are given by $v_{t}\left(\theta_{1}, \xi^{S^{t}}\right)-\frac{1-F\left(\theta_{1}\right)}{f\left(\theta_{1}\right)} \frac{\partial v_{t}\left(\theta_{1}, \xi^{S^{t}}\right)}{\partial \theta_{1}}$. These values depend only on first period distribution $F$ and not on $G$. This reflects the fact that the seller commits to the contract at period 1. The distributions $G$ however do affect the optimal and efficient contract as they represent the value of experimentation. 
Finally, we need to show that we can find prices so that the solution to the relaxed problem can be implemented in an incentive compatible way. This is summarized by the following proposition the proof of which is in the appendix.

Proposition 4. The quantity schedules of the incentive compatible, individually rational seller optimal contract coincide with that of the relaxed problem stated in proposition (3). In other words, we can find prices to implement the quantity schedules of (3) such that the resulting mechanism gives both the buyer and the seller the same utility and is incentive compatible.

\section{A Two Period Example}

In this section we discuss a simple example of the above model. This example highlights some of the key features of the model. We assume that initial valuation $\theta_{1}$ is drawn from the uniform $\mathbb{U}[0,1]$ distribution (which is log concave and hence satisfies the monotone hazard rate condition). If the buyer receives the object then her signal $\xi_{2}$ is drawn from the $\mathbb{U}[-1,1]$ distribution. The value function $v_{2}$ is assumed to be additive and is given by $v_{2}\left(\theta_{1}, \xi_{2}\right)=\theta_{1}+\xi_{2}$. Thus the signal can both decrease or increase the value in period 2; in particular, it can drive the buyer's valuation below 0 . Linear $v_{2}$ clearly satisfies the assumptions we need for our results. We can now derive the optimal allocations:

$$
\begin{aligned}
q_{1}\left(\theta_{1}\right) & = \begin{cases}1 & \text { if } \theta_{1} \geq \theta_{l} \\
0 & \text { otherwise }\end{cases} \\
q_{2}\left(\theta_{1}, \xi_{2}\right) & = \begin{cases}1 & \text { if } \xi_{2} \geq 1-2 \theta_{1} \\
0 & \text { otherwise }\end{cases}
\end{aligned}
$$

and the corresponding optimal prices

$$
\begin{aligned}
p_{1}\left(\theta_{1}\right) & = \begin{cases}\theta_{l}+\frac{\delta}{2}\left[\theta_{l}^{2}+\theta_{1}^{2}\right] & \text { if } \theta_{1} \geq \theta_{l} \\
0 & \text { otherwise }\end{cases} \\
p_{2}\left(\theta_{1}, \xi_{2}\right) & = \begin{cases}1-\theta_{1} & \text { if } \xi_{2} \geq 1-2 \theta_{1} \\
0 & \text { otherwise }\end{cases} \\
\text { where } \theta_{l} & =\frac{\sqrt{1+\delta}-1}{\delta}
\end{aligned}
$$

It is interesting to note that the buyer with the lowest value who receives the object (the buyer with value $\theta_{l}$ ) pays more for the object than her value. Hence, in the optimal mechanism the seller does not offer a low introductory price in period 1 which allows everyone to revise their valuation for the product. Rather, he does not offer the product to buyers of lowest valuations and instead levies a charge for the consumption experience. Of course, it is possible to construct a different example of the model such that $\theta_{l}=\underline{\theta}$, so that the seller sells the object to the entire market. However, even in this case the buyer with the lowest valuation (who gets the object) will pay at least her value in period 1 to try the object. This behavior is individually rational because she has 
the option to terminate the contract at period 2, hence, expects at least a weak increase in her valuation in period 2 and as a result her expected utility in period 1 is non-negative.

Notice, also that the cutoff value $\theta_{l}$ is decreasing in $\delta$. When $\delta$ approaches $0, \theta_{l}$ approaches $1 / 2$ which is the cutoff value of the static optimal auction of Myerson (1981). This is because when $\delta$ approaches 0 , neither the buyer nor the seller (initially) cares about the next period and the seller tries to extract as much revenue as possible at $t=1$. As $\delta$ increases, the cutoff value $\theta_{l}$ begins to fall. This reflects the fact that the seller values revenue from future participation and also the fact that the buyer benefits more from experimentation.

The price charged by the seller in period 1 is strictly increasing in the announced valuation. The price in period 2 is independent of the signal $\xi_{2}$ and is decreasing in the $\theta_{1}$ announced by the buyer in period 1. This mechanism is intuitively incentive compatible due to the following reason. For buyers with high valuation, there is a high probability that they would like to purchase the product in period 2 after observing $\xi_{2}$. Announcing a high valuation guarantees them a relatively low price tomorrow as the price in period 2 depends only on the $\theta_{1}$ announced in period 1 . Buyers with low valuations prefer to consume the product in period 1 for a low price and as a result face a relatively higher price in period 2. However, since they feel it is unlikely that they will like the product once they try it, they are content simply to try it at a low price.

The optimal contract is clearly inefficient. In this setting an efficient contract would allocate the object to the buyer with probability 1 if her valuation $\theta_{1}$ is strictly greater than 0 in period 1 . In period 2, the efficient contract would allocate the object to the buyer with probability 1 , if her revised valuation $v_{2}\left(\theta_{1}, \xi_{2}\right)$ is strictly greater than 0 . In contrast, the optimal contract does not allocate the object the buyers with valuation below $\theta_{l}$ in period 1 , and to buyers with valuation $v_{2}\left(\theta_{1}, \xi_{2}\right)$ less than $1-\theta_{1}$ in period 2. The intuition for this is identical to that of the optimal auction in a static setting.

\section{Independent SHOCKS}

In this section we briefly address the assumption of independent shocks. We assumed that the distribution $G$ of $\xi_{t}$ is independent of the distribution $F$ of $\theta_{1}$. This assumption is actually less restrictive than it may initially appear. We discuss the implication of this assumption when $T=2$ as discussed in Eso and Szentes (2007). Again restricting the discussion to 2 periods makes it easy to interpret the nature of the assumption. The intuition in this section has been generalized to $T$ periods by PST.

Let $H\left(\theta_{2} \mid \theta_{1}\right)$ be the distribution of period 2 values conditional on realized period 1 value and let $h\left(\theta_{2} \mid \theta_{1}\right)$ be the associated pdf. The following is an insightful result from Eso and Szentes (2007).

Lemma 6.1 (Eso and Szentes (2007)). Suppose the distribution $H\left(\theta_{2} \mid \theta_{1}\right)$ of $\theta_{2}$ conditional on $\theta_{1}$ is continuous and strictly increasing in $\theta_{2}$. Then there exists a real valued function $v$ and a random 
variable $\xi_{2}$ where $\xi_{2}$ and $\theta_{1}$ are independent such that

$$
\theta_{2}=v_{2}\left(\theta_{1}, \xi_{2}\right)
$$

The intuition of this result stems from the following observation. We can define $\xi_{2}=H\left(\theta_{2} \mid \theta_{1}\right)$. Then $\xi_{2}$ is distributed uniformly $\mathbb{U}[0,1]$ and is independent of $\theta_{1}$. The value function $v_{2}$ can then be defined as $v_{2}\left(\theta_{1}, \xi_{2}\right)=H_{\theta_{1}}^{-1}\left(\xi_{2}\right)$ where $H_{\theta_{1}}^{-1}\left(\xi_{2}\right)=\theta_{2}$ such that $H\left(\theta_{2} \mid \theta_{1}\right)=\xi_{2}$. The function is invertible because we assumed $H$ was continuous and strictly increasing. So in general, this assumption is not restrictive at all.

However, for our results we require the additional assumptions A2-A4 on the value function $v_{2}$ (assumption A5 is not required when $T=2$ ). Hence, we need additional assumptions on the stochastic process $H$. The following assumptions also derived by Eso and Szentes (2007), on the stochastic process are sufficient for our results.

A6 $H\left(\theta_{2} \mid \theta_{1}\right)$ is continuous and strictly increasing in $\theta_{2}$.

A7 For $\theta_{1}^{\prime}>\theta_{1}, H\left(\theta_{2} \mid \theta_{1}^{\prime}\right)$ first order stochastically dominates $H\left(\theta_{2} \mid \theta_{1}\right)$ or in other words $\partial H\left(\theta_{2} \mid \theta_{1}\right) / \partial \theta_{1}<0$.

A8 $\frac{\partial H\left(\theta_{2} \mid \theta_{1}\right) / \partial \theta_{1}}{h\left(\theta_{2} \mid \theta_{1}\right)}$ is increasing in $\theta_{2}$.

A9 $\frac{\partial H\left(\theta_{2} \mid \theta_{1}\right) / \partial \theta_{1}}{h\left(\theta_{2} \mid \theta_{1}\right)}$ is increasing in $\theta_{1}$.

PST show that for arbitrary $T$ periods, the sufficient condition to satisfy assumptions $A 2-A 5$, is first order stochastic dominance and that $\frac{\partial H_{t}\left(\theta_{t} \mid \theta_{t-1}\right) / \partial \theta_{t-1}}{h_{t}\left(\theta_{t} \mid \theta_{t-1}\right)}$ is increasing in both $\theta_{t}$ and $\theta_{t-1}$, where $H_{t}$ is the conditional distribution of $\theta_{t}$ given $\theta_{t-1}$. These assumptions are satisfied by many stochastic processes. Therefore, while the assumption of independent shocks does result in a loss of generality, we do not find it to be excessively restrictive and hence, assuming independent shocks is not as severe a constraint as it may have initially seemed.

\section{EXTENSIONS}

The model presented in this paper can be extended in a fairly straightforward way to the case of $N$ buyers competing for a single object in each period, over the course of $T$ periods. Only the buyer who wins the object in a given period gets to consume the object and hence update her valuation through her consumption experience. At period $t$, the optimal contract will award the object the buyer with the highest expected discounted sum of virtual flow values. The closed form solutions though are extremely complicated as we need to consider a large set of possible histories. It is possible for a buyer to win an object in period $t$, receive a bad signal and lose the object in period $t+1$, only to win it again in period $t+2$ after the buyer who won in $t+1$ also receives a bad signal. This is in contrast to the case of the single buyer which is simply a stopping rule.

Sponsored search in particular and advertising in general is an example of a real life situation of such an auction model with learning. Advertisements, by their very nature, are products where the advertiser cannot accurately asses the value of advertising before placing the ad. Hence, the 
expected value of an ad to an advertiser could potentially differ drastically before and after placing the ad. Sponsored search auctions are dynamic in nature, with the competing advertisers constantly updating their valuations over time. There is a current and ongoing economics literature which studies sponsored search (see Edelman et al. (2007), Varian (2007), Borgers et al. (2007) and Nazerzadeh et al. (2007) to name but a few papers). Of course, while our model captures the learning aspect of advertising it ignores the fact that advertisers often have more than one option in each period. In sponsored search auctions, bidders who lose the auction for the best link position can potentially win the second best position and so on. A model that captures both this aspect and learning is a subject of future research.

Finally, it is also possible to extend the model in such a way so that the distribution of shocks $G$ is not the same in all periods. The analysis will go through identically if we assume that the first time the buyer consumes the product her signal comes from distribution $G_{1}$, the second time from distribution $G_{2}$ and so on. Notice that this is not the same as saying that if she consumes the product in period $t-1$ she will receive a signal from distribution $G_{t}$. If the distribution of the signals were a function of the time period as opposed to the number of times the buyer consumes the object then the optimal contract need not be a stopping rule. Consider the former case, where distribution $G_{t}$ has a very low mean and $G_{t+1}$ has a very high mean. At period $t-1$, it may be optimal for the seller not to allocate the object to the buyer as then she will probably receive a low signal from $G_{t}$ and instead allocate it to her in period $t$ allowing her to receive the high signal from $G_{t+1}$ and hence allowing the seller to extract more surplus.

\section{Appendix A. Deriving the Envelope Condition}

We now explicitly derive the envelope condition at period 1 . Consider the utility to the buyer from reporting truthfully at period 1 .

$U_{1}\left(\theta_{1}\right)=\theta_{1} q_{1}\left(\theta_{1}\right)-p_{1}\left(\theta_{1}\right)+$

$$
\begin{gathered}
\sum_{t=2}^{T} \sum_{C^{t-1} \in \mathscr{C}^{t-1}} \delta^{t} \int_{\Xi_{2}} \ldots \int_{\Xi_{T}} \mu_{q}\left(C^{t-1}\right)\left[q_{t}^{C^{t-1}}\left(\theta_{1}, \xi^{C^{t-1}}\right) v_{t}^{C^{t-1}}\left(\theta_{1}, \xi^{C^{t-1}}\right)-p_{t}^{C^{t-1}}\left(\theta_{1}, \xi^{C^{t-1}}\right)\right] \\
d G\left(\xi_{2}\right) \ldots d G\left(\xi_{T}\right)
\end{gathered}
$$

where $\mu_{q}\left(C^{t-1}\right)$ is the probability that history of consumption $C^{t-1}$ occurs as a result of contract $q$ and reporting $\theta_{1}$ in period 1 and truthful reporting thereafter. If instead she reports $\theta_{1}^{\prime}$ in period 1 and subsequently reports truthfully she gets

$$
\begin{aligned}
& \theta_{1} q_{1}\left(\theta_{1}^{\prime}\right)-p_{1}\left(\theta_{1}^{\prime}\right)+ \\
& \sum_{t=2}^{T} \sum_{C^{t-1} \in \mathscr{C}^{t-1}} \delta^{t} \int_{\Xi_{2}} \ldots \int_{\Xi_{T}} \mu_{q}^{\prime}\left(C^{t-1}\right)\left[q_{t}^{C^{t-1}}\left(\theta_{1}^{\prime}, \xi^{C^{t-1}}\right) v_{t}^{C^{t-1}}\left(\theta_{1}, \xi^{C^{t-1}}\right)-p_{t}^{C^{t-1}}\left(\theta_{1}^{\prime}, \xi^{C^{t-1}}\right)\right] \\
& d G\left(\xi_{2}\right) \ldots d G\left(\xi_{T}\right)
\end{aligned}
$$

where $\mu_{q}^{\prime}\left(C^{t-1}\right)$ is the probability that history of consumption $C^{t-1}$ occurs as a result of contract $q$ and reporting $\theta_{1}^{\prime}$ in period 1 and truthful reporting thereafter. Notice that in the above expression 
the buyer does not report strategically after period 1 . There is no reason to believe that truthtelling is optimal after the buyer has misreported in the first period. Incentive compatibility implies that the $(11) \geq(12)$. Rearranging

$$
\begin{aligned}
U_{1}\left(\theta_{1}\right)-U_{1}\left(\theta_{1}^{\prime}\right) \geq & \left(\theta_{1}-\theta_{1}^{\prime}\right) q_{1}\left(\theta_{1}^{\prime}\right)+ \\
& \sum_{t=2}^{T} \sum_{C^{t-1} \in \mathscr{C}^{t-1}} \delta^{t} \int_{\Xi_{2}} \ldots \int_{\Xi_{T}} \mu_{q}^{\prime}\left(C^{t-1}\right) q_{t}^{C^{t-1}}\left(\theta_{1}^{\prime}, \xi^{C^{t-1}}\right)\left[v_{t}^{C^{t-1}}\left(\theta_{1}, \xi^{C^{t-1}}\right)\right. \\
& \left.-v_{t}^{C^{t-1}}\left(\theta_{1}^{\prime}, \xi^{C^{t-1}}\right)\right] d G\left(\xi_{2}\right) \ldots d G\left(\xi_{T}\right)
\end{aligned}
$$

Exchanging the roles of $\theta_{1}$ and $\theta_{1}^{\prime}$ we get

$$
\begin{aligned}
& U_{1}\left(\theta_{1}\right)-U_{1}\left(\theta_{1}^{\prime}\right) \leq\left(\theta_{1}-\theta_{1}^{\prime}\right) q_{1}\left(\theta_{1}\right)+ \\
& \sum_{t=2}^{T} \sum_{C^{t-1} \in \mathscr{C}^{t-1}} \delta^{t} \int_{\Xi_{2}} \ldots \int_{\Xi_{T}} \mu_{q}\left(C^{t-1}\right) q_{t}^{C^{t-1}}\left(\theta_{1}, \xi^{C^{t-1}}\right)\left[v_{t}^{C^{t-1}}\left(\theta_{1}, \xi^{C^{t-1}}\right)\right. \\
&\left.-v_{t}^{C^{t-1}}\left(\theta_{1}^{\prime}, \xi^{C^{t-1}}\right)\right] d G\left(\xi_{2}\right) \ldots d G\left(\xi_{T}\right)
\end{aligned}
$$

Dividing both sides of (13) and (14) by $\theta_{1}-\theta_{1}^{\prime}$ and taking the limit $\theta_{1}^{\prime} \rightarrow \theta_{1}$ we get the required envelope condition.

$$
\begin{aligned}
& \frac{d U_{1}\left(\theta_{1}\right)}{d \theta_{1}}= q_{1}\left(\theta_{1}\right)+ \\
& \sum_{t=2}^{T} \sum_{C^{t-1} \in \mathscr{C}^{t-1}} \delta^{t} \int_{\Xi_{2}} \ldots \int_{\Xi_{T}} \mu_{q}\left(C^{t-1}\right)\left[q_{t}^{C^{t-1}}\left(\theta_{1}, \xi^{C^{t-1}}\right) \frac{\partial v_{t}^{C^{t-1}}\left(\theta_{1}, \xi^{C^{t-1}}\right)}{\partial \theta_{1}}\right] \\
& d G\left(\xi_{2}\right) \ldots d G\left(\xi_{T}\right)
\end{aligned}
$$

\section{Appendix B. Proof of Incentive Compatibility}

Proof. The optimal contract has $q_{t}^{C^{t}}=0$ for all $C^{t} \neq S^{t}$. Clearly, it must also be the case that $p_{t}^{C^{t}}=0$ for all $C^{t} \neq S^{t}$. Hence, revenues for the seller and utility for the buyer will only contain the terms $q_{t}^{S^{t}}, p_{t}^{S^{t}}$. In this proof we will refer to $q_{t}^{S^{t}}, p_{t}^{S^{t}}$ simply as $q_{t}, p_{t}$. This makes the proof considerably more intuitive as we have to deal with less notation. We need to show that there are prices which make the solution to the relaxed problem incentive compatible. We first need to check the following monotonicity condition. This condition is the following.

(1) Quantity schedules $q_{t}$ are nondecreasing in each argument.

(2) For any $t \geq 2$ and any $s \geq t+1$ and any $\left(\theta_{1}, \xi^{t-1}, \xi_{t}, \xi_{t+1}\right)$ and $\left(\theta_{1}, \xi^{t-1}, \tilde{\xi}_{t}, \tilde{\xi}_{t+1}\right)$ such that $v_{t+1}\left(\theta_{1}, \xi^{t-1}, \xi_{t}, \xi_{t+1}\right)=v_{t+1}\left(\theta_{1}, \xi^{t-1}, \tilde{\xi}_{t}, \tilde{\xi}_{t+1}\right)$

$$
q_{s}\left(\theta_{1}, \xi^{t-1}, \xi_{t}, \xi_{t+1}, \ldots, \xi_{s}\right) \leq(\geq) q_{s}\left(\theta_{1}, \xi^{t-1}, \tilde{\xi}_{t}, \tilde{\xi}_{t+1}, \ldots, \xi_{s}\right)
$$

if and only if $\xi_{t} \leq(\geq) \tilde{\xi}_{t}$ and vice versa. 
(3) Similarly, for any $\left(\theta_{1}, \xi_{2}\right)$ and $\left(\tilde{\theta}_{1}, \tilde{\xi}_{2}\right)$ such that $v_{2}\left(\theta_{1}, \xi_{2}\right)=v_{2}\left(\tilde{\theta}_{1}, \tilde{\xi}_{2}\right)$,

$$
q_{s}\left(\theta_{1}, \xi_{2}, \ldots, \xi_{s}\right) \leq(\geq) q_{s}\left(\tilde{\theta}_{1}, \tilde{\xi}_{2}, \ldots, \xi_{s}\right)
$$

if and only if $\theta_{1} \leq(\geq) \tilde{\theta}_{1}$.

It is straightforward to observe that A1-A4 imply that the quantity schedules are nondecreasing in each argument. Hence, we only need to show monotonicity properties (2) and (3) as above. We will show property (2) and (3) will follow similarly. We do this by backward induction on the quantity schedules. We will show the $\xi_{t} \leq \tilde{\xi}_{t}$ case and the $\xi_{t} \geq \tilde{\xi}_{t}$ case is analogous. Pavan (2007) shows that the assumptions A1-A5 imply that

$$
\begin{aligned}
& v_{s}\left(\theta_{1}, \xi^{t-1}, \xi_{t}, \xi_{t+1}, \ldots, \xi_{s}\right)-\frac{\partial v_{s}\left(\theta_{1}, \xi^{t-1}, \xi_{t}, \xi_{t+1}, \ldots, \xi_{s}\right)}{\partial \theta_{1}} \frac{1-F\left(\theta_{1}\right)}{f\left(\theta_{1}\right)} \leq \\
& v_{s}\left(\theta_{1}, \xi^{t-1}, \tilde{\xi}_{t}, \tilde{\xi}_{t+1}, \ldots, \xi_{s}\right)-\frac{\partial v_{s}\left(\theta_{1}, \xi^{t-1}, \tilde{\xi}_{t}, \tilde{\xi}_{t+1}, \ldots, \xi_{s}\right)}{\partial \theta_{1}} \frac{1-F\left(\theta_{1}\right)}{f\left(\theta_{1}\right)}
\end{aligned}
$$

whenever $v_{t+1}\left(\theta_{1}, \xi^{t-1}, \xi_{t}, \xi_{t+1}\right)=v_{t+1}\left(\theta_{1}, \xi^{t-1}, \tilde{\xi}_{t}, \tilde{\xi}_{t+1}\right)$ and $\xi_{t} \leq \tilde{\xi}_{t}$. In particular this implies that the above holds for $s=T$ and hence $q^{T}$ satisfies the monotonicity condition. Now since

$$
\begin{aligned}
& v_{T}\left(\theta_{1}, \xi^{t-1}, \xi_{t}, \xi_{t+1}, \ldots, \xi_{T}\right)-\frac{\partial v_{T}\left(\theta_{1}, \xi^{t-1}, \xi_{t}, \xi_{t+1}, \ldots, \xi_{T}\right)}{\partial \theta_{1}} \frac{1-F\left(\theta_{1}\right)}{f\left(\theta_{1}\right)} \leq \\
& v_{T}\left(\theta_{1}, \xi^{t-1}, \tilde{\xi}_{t}, \tilde{\xi}_{t+1}, \ldots, \xi_{T}\right)-\frac{\partial v_{T}\left(\theta_{1}, \xi^{t-1}, \tilde{\xi}_{t}, \tilde{\xi}_{t+1}, \ldots, \xi_{T}\right)}{\partial \theta_{1}} \frac{1-F\left(\theta_{1}\right)}{f\left(\theta_{1}\right)} \text { and } \\
& v_{T-1}\left(\theta_{1}, \xi^{t-1}, \xi_{t}, \xi_{t+1}, \ldots, \xi_{T-1}\right)-\frac{\partial v_{T-1}\left(\theta_{1}, \xi^{t-1}, \xi_{t}, \xi_{t+1}, \ldots, \xi_{T-1}\right)}{\partial \theta_{1}} \frac{1-F\left(\theta_{1}\right)}{f\left(\theta_{1}\right)} \leq \\
& v_{T-1}\left(\theta_{1}, \xi^{t-1}, \tilde{\xi}_{t}, \tilde{\xi}_{t+1}, \ldots, \xi_{T-1}\right)-\frac{\partial v_{T-1}\left(\theta_{1}, \xi^{t-1}, \tilde{\xi}_{t}, \tilde{\xi}_{t+1}, \ldots, \xi_{T-1}\right)}{\partial \theta_{1}} \frac{1-F\left(\theta_{1}\right)}{f\left(\theta_{1}\right)} \text { and } \\
& q_{T}\left(\theta_{1}, \xi^{t-1}, \xi_{t}, \xi_{t+1}, \ldots, \xi_{T}\right) \leq q_{T}\left(\theta_{1}, \xi^{t-1}, \tilde{\xi}_{t}, \tilde{\xi}_{t+1}, \ldots, \xi_{T}\right)
\end{aligned}
$$

This implies that $q_{T-1}\left(\theta_{1}, \xi^{t-1}, \xi_{t}, \xi_{t+1}, \ldots, \xi_{T-1}\right) \leq q_{T-1}\left(\theta_{1}, \xi^{t-1}, \tilde{\xi}_{t}, \tilde{\xi}_{t+1}, \ldots, \xi_{T-1}\right)$. This argument can be further inducted backwards to show that the monotonicity conditions are satisfied by all $q_{t}$.

We are now in a position to derive prices so that the resulting contract is incentive compatible. From the definition of buyer utilities we can inductively derive the transfers. In other words, we use the following equations

$$
U_{T}\left(\theta_{1}, \xi^{T}\right)=v_{T}\left(\theta_{1}, \xi^{T}\right) q_{T}\left(\theta_{1}, \xi^{T}\right)-p_{T}\left(\theta_{1}, \xi^{T}\right)
$$

and

$$
U_{t}\left(\theta_{1}, \xi^{t}\right)=v_{t}\left(\theta_{1}, \xi^{t}\right) q_{t}\left(\theta_{1}, \xi^{t}\right)-p_{t}\left(\theta_{1}, \xi^{t}\right)+\delta q_{t}\left(\theta_{1}, \xi^{t}\right) \int_{\Xi_{t+1}} U_{t+1}\left(\theta_{1}, \xi^{t}, \xi_{t+1}\right) d G\left(\xi_{t+1}\right)
$$

We first observe that if the buyer reported truthfully up to period $T$ it is immediate that she will report $\xi_{T}$ truthfully as well. This is because the envelope condition for the final period is necessary and sufficient for incentive compatibility (see Myerson (1981)). However, as we have pointed out, for any $t<T$, the envelope condition is merely necessary for incentive compatibility. 
We proceed inductively by choosing an arbitrary $t<T$ and assuming that the contract is incentive compatible for periods $s>t$. This implies that if the buyer reports truthfully till period $t$, then the best she can do is to report truthfully for any subsequent period. Now we show that it is optimal for the buyer to report truthfully at period $t$ having reported truthfully till period $t-1$. If instead, she reports $\hat{\xi}_{t} \neq \xi_{t}$ in period $t$, she gets

$$
\begin{aligned}
\hat{U}_{t}\left(\theta_{1}, \xi^{t-1}, \xi_{t} ; \theta_{1}, \xi^{t-1}, \hat{\xi}_{t}\right)= & v_{t}\left(\theta_{1}, \xi^{t}\right) q_{t}\left(\theta_{1}, \xi^{t-1}, \hat{\xi}_{t}\right)-p_{t}\left(\theta_{1}, \hat{\xi}^{t}\right)+ \\
& \delta q_{t}\left(\theta_{1}, \xi^{t-1}, \hat{\xi}_{t}\right) \int_{\Xi_{t+1}} \hat{U}_{t+1}\left(\theta_{1}, \xi^{t}, \xi_{t+1} ; \theta, \xi_{t-1}, \hat{\xi}_{t}\right) d G\left(\xi_{t+1}\right)
\end{aligned}
$$

Now note that

$$
\begin{aligned}
& \hat{U}_{t}\left(\theta_{1}, \xi^{t-1}, \xi_{t} ; \theta_{1}, \xi^{t-1}, \hat{\xi}_{t}\right)= \\
& U_{t}\left(\theta_{1}, \xi^{t-1}, \hat{\xi}_{t}\right)+\left[v_{t}\left(\theta_{1}, \xi^{t}\right)-v_{t}\left(\theta_{1}, \hat{\xi}^{t}\right)\right] q_{t}\left(\theta_{1}, \xi^{t-1}, \hat{\xi}_{t}\right)+ \\
& \delta q_{t}\left(\theta_{1}, \xi^{t-1}, \hat{\xi}_{t}\right) \int_{\Xi_{t+1}}\left[\hat{U}_{t+1}\left(\theta_{1}, \xi^{t}, \xi_{t+1} ; \theta, \xi_{t-1}, \hat{\xi}_{t}\right)-U_{t+1}\left(\theta_{1}, \xi^{t-1}, \hat{\xi}_{t}, \xi_{t+1}\right)\right] d G\left(\xi_{t+1}\right)
\end{aligned}
$$

Hence we can rewrite the incentive constraint as

$$
\begin{aligned}
& U_{t}\left(\theta_{1}, \xi^{t-1}, \xi_{t}\right)-U_{t}\left(\theta_{1}, \xi^{t-1}, \hat{\xi}_{t}\right) \geq \\
& \int_{\hat{\xi}_{T}}^{\xi_{t}}\left[\frac{\partial v_{t}\left(\theta_{1}, \xi^{t-1}, \tilde{\xi}_{t}\right)}{\partial \tilde{\xi}_{t}} q_{t}\left(\theta_{1}, \xi^{t-1}, \hat{\xi}_{t}\right)\right] d \tilde{\xi}+ \\
& \delta q_{t}\left(\theta_{1}, \xi^{t-1}, \hat{\xi}_{t}\right) \int_{\hat{\xi}_{T}}^{\xi_{t}}\left[\int_{\Xi_{t+1}} \frac{\partial \hat{U}_{t+1}\left(\theta_{1}, \xi^{t-1}, \tilde{\xi}_{t}, \xi_{t+1} ; \theta, \xi_{t-1}, \hat{\xi}_{t}\right)}{\partial \tilde{\xi}_{t}} d G\left(\xi_{t+1}\right)\right] d \tilde{\xi}_{t}
\end{aligned}
$$

Taking the difference between utilities and using the envelope theorem at period $t$ yields

$$
\begin{aligned}
& U_{t}\left(\theta_{1}, \xi^{t-1}, \xi_{t}\right)-U_{t}\left(\theta_{1}, \xi^{t-1}, \hat{\xi}_{t}\right)= \\
& \int_{\hat{\xi}_{t}}^{\xi_{t}}\left\{\frac{\partial v_{t}\left(\theta_{1}, \xi^{t-1}, \tilde{\xi}_{t}\right)}{\partial \tilde{\xi}_{t}} q_{t}\left(\theta_{1}, \xi^{t-1}, \tilde{\xi}_{t}\right)+\right. \\
& q_{t}\left(\theta_{1}, \xi^{t-1}, \tilde{\xi}_{t}\right) \int_{\Xi_{t+1}} \ldots \int_{\Xi_{T}}\left[\delta \frac{\partial v_{t+1}\left(\theta_{1}, \xi^{t-1}, \tilde{\xi}_{t}, \xi_{t+1}\right)}{\partial \tilde{\xi}_{t}} q_{t+1}\left(\theta_{1}, \xi^{t-1}, \tilde{\xi}_{t}, \xi_{t+1}\right)+\cdots+\right. \\
& \left.\left.\delta^{T-t} \frac{\partial v_{T}\left(\theta_{1}, \xi^{t-1}, \tilde{\xi}_{t}, \xi_{t+1}, \ldots, \xi_{T}\right)}{\partial \tilde{\xi}_{t}}\left(\prod_{s=t+1}^{T} q_{s}\left(\theta_{1}, \xi^{t-1}, \tilde{\xi}_{t}, \ldots, \xi_{s}\right)\right)\right] d G\left(\xi_{t+1}\right) \ldots d G\left(\xi_{T}\right)\right\} d \tilde{\xi}_{t}
\end{aligned}
$$

Similarly the envelope theorem at period $t+1$ yields

$$
\begin{aligned}
& \frac{\partial \hat{U}_{t+1}\left(\theta_{1}, \xi^{t-1}, \tilde{\xi}_{t}, \xi_{t+1} ; \theta_{1}, \xi^{t-1}, \hat{\xi}_{t}\right)}{\partial \tilde{\xi}_{t}}= \\
& \frac{\partial v_{t+1}\left(\theta_{1}, \xi^{t-1}, \tilde{\xi}_{t}, \xi_{t+1}\right)}{\partial \tilde{\xi}_{t}} q_{t+1}\left(\theta_{1}, \xi^{t-1}, \hat{\xi}_{t}, \hat{\xi}_{t+1}^{*}\left(\tilde{\xi}_{t}\right)\right)+ \\
& \delta q_{t+1}\left(\theta_{1}, \xi^{t-1}, \hat{\xi}_{t}, \hat{\xi}_{t+1}^{*}\left(\tilde{\xi}_{t}\right)\right) \int_{\Xi_{t+2}} \frac{\partial \hat{U}_{t+2}\left(\theta_{1}, \xi^{t-1}, \tilde{\xi}_{t}, \xi_{t+1}, \xi_{t+2} ; \theta_{1}, \xi^{t-1}, \hat{\xi}_{t}, \hat{\xi}_{t+1}^{*}\left(\tilde{\xi}_{t}\right)\right)}{\partial \tilde{\xi}_{t}} d G\left(\xi_{t+2}\right)
\end{aligned}
$$

where $\hat{\xi}_{t+1}^{*}\left(\tilde{\xi}_{t}\right)$ is the optimal report in period $t+1$ given that she reported $\hat{\xi}_{t}$ in period $t . \hat{\xi}_{t+1}^{*}\left(\tilde{\xi}_{t}\right)$ is the implicit solution to the equation $v_{t+1}\left(\theta_{1}, \xi^{t-1}, \hat{\xi}_{t}, \hat{\xi}_{t+1}^{*}\right)=v_{t+1}\left(\theta_{1}, \xi^{t-1}, \tilde{\xi}_{t}, \xi_{t+1}\right)$. The reason 
for this is the following. Assume that the true signals observed by the buyer up to period $t+1$ are $\theta_{1}, \xi^{t-1}, \hat{\xi}_{t}, \hat{\xi}_{t+1}^{*}\left(\tilde{\xi}_{t}\right)$. Then if the buyer reports truthfully up to period $t$ it must be optimal for the buyer to truthfully report $\hat{\xi}_{t+1}^{*}\left(\tilde{\xi}_{t}\right)$ in period $t+1$ by the inductive hypothesis. But from the definition of $\hat{\xi}_{t+1}^{*}\left(\tilde{\xi}_{t}\right)$, the buyer's valuation in period $t+1$ is $v_{t+1}\left(\theta_{1}, \xi^{t-1}, \hat{\xi}_{t}, \hat{\xi}_{t+1}^{*}\left(\tilde{\xi}_{t}\right)\right)=v_{t+1}\left(\theta_{1}, \xi^{t-1}, \tilde{\xi}_{t}, \xi_{t+1}\right)$. Hence, when the buyer has this value it is optimal for her to report $\hat{\xi}_{t+1}^{*}\left(\tilde{\xi}_{t}\right)$ when she misreports $\hat{\xi}_{t}$ in period $t$.

We can now plug (17) into the right hand side of (15) and we get

$$
\begin{aligned}
& \int_{\hat{\xi}_{T}}^{\xi_{t}}\left[\frac{\partial v_{t}\left(\theta_{1}, \xi^{t-1}, \tilde{\xi}_{t}\right)}{\partial \tilde{\xi}_{t}} q_{t}\left(\theta_{1}, \xi^{t-1}, \hat{\xi}_{t}\right)+\right. \\
& q_{t}\left(\theta_{1}, \xi^{t-1}, \hat{\xi}_{t}\right) \int_{\Xi_{t+1}} \ldots \int_{\Xi_{T}}\left\{\delta \frac{\partial v_{t+1}\left(\theta_{1}, \xi^{t-1}, \tilde{\xi}_{t}, \xi_{t+1}\right)}{\partial \tilde{\xi}_{t}} q_{t+1}\left(\theta_{1}, \xi^{t-1}, \hat{\xi}_{t}, \hat{\xi}_{t+1}^{*}\left(\tilde{\xi}_{t}\right)\right)+\right. \\
& \delta^{2} \frac{\partial v_{t+2}\left(\theta_{1}, \xi^{t-1}, \tilde{\xi}_{t}, \xi_{t+1}, \xi_{t+2}\right)}{\partial \tilde{\xi}_{t}} q_{t+1}\left(\theta_{1}, \xi^{t-1}, \hat{\xi}_{t}, \hat{\xi}_{t+1}^{*}\left(\tilde{\xi}_{t}\right)\right) q_{t+2}\left(\theta_{1}, \xi^{t-1}, \hat{\xi}_{t}, \hat{\xi}_{t+1}^{*}\left(\tilde{\xi}_{t}\right), \xi_{t+2}\right)+\cdots+ \\
& \left.\left.\delta^{T-t} \frac{\partial v_{T}\left(\theta_{1}, \xi^{t-1}, \tilde{\xi}_{t}, \xi_{t+1}, \ldots, \xi_{T}\right)}{\partial \tilde{\xi}_{t}}\left(\prod_{s=t+1}^{T} q_{s}\left(\theta_{1}, \xi^{t-1}, \hat{\xi}_{t}, \hat{\xi}_{t+1}^{*}\left(\tilde{\xi}_{t}\right), \ldots, \xi_{s}\right)\right)\right\} d G\left(\xi_{t+1}\right) \ldots d G\left(\xi_{T}\right)\right] d \tilde{\xi}_{t}
\end{aligned}
$$

We can compare the above expression with (16). The monotonicity property ensures that the incentive compatibility condition (15) is satisfied and this completes the proof.

\section{REFERENCES}

[1] S. Athey and I. Segal. An efficient dynamic mechanism. Working Paper, 2007.

[2] D. Baron and D. Besanko. Regulation and information in a continuing relationship. Information Economics and Policy, 1984.

[3] M. Battaglini. Long-term contracting with markovian consumers. American Economic Review, 95:637-658, 2005.

[4] D. Bergemann and J. Valimaki. Bandit problems. Cowles Foundation Discussion Paper, 2006.

[5] D. Bergemann and J. Valimaki. Dynamic marginal contribution mechanism. Cowles Foundation Discussion Paper, 2007.

[6] T. Borgers, I. Cox, M. Pesendorfer, and V. Petricek. Equilibrium bids in sponsored search auctions: Theory and evidence. Working Paper, 2007.

[7] P. Courty and H. Li. Sequential screening. Review of Economic Studies, 2000.

[8] B. Edelman, M. Ostrovsky, and M. Schwarz. Internet advertising and the generalized second-price auction: Selling billions of dollars worth of keywords. American Economic Review, 97:242-259, 2007.

[9] P. Eso and B. Szentes. Optimal information disclosure in auctions and the handicap auction. Review of Economic Studies, 74:705-731, 2007.

[10] R. Myerson. Optimal auction design. Mathematics of Operations Research, 1981.

[11] H. Nazerzadeh, A. Saberi, and R. Vohra. Dynamic cost-per-action mechanisms and applications to online advertising. Working Paper, 2007.

[12] A. Pavan. Long-term contracting in a changing world. Working Paper, 2007.

[13] A. Pavan, I. Segal, and J. Toikka. Dynamic mechanism design: Revenue equivalence, profit maximization, and information disclosure. Working Paper, 2008.

[14] H. Varian. Position auctions. International Journal of Industrial Organization, 25:1163-1178, 2007. 